\title{
Phonon exchange in dilute Fermi-Bose mixtures: tailoring the Fermi-Fermi interaction.
}

\author{
M.J. Bijlsma, B.A. Heringa, and H.T.C. Stoof \\ Institute for Theoretical Physics, University of Utrecht, \\ Princetonplein 5, 3584 CC Utrecht, The Netherlands
}

\begin{abstract}
We consider a mixture of a single-component Bose gas and a two-component Fermi gas at temperatures where the Bose gas is almost fully condensed. In such a mixture, two fermionic atoms can interact with each other by exchanging a phonon that propagates through the Bose condensate. We calculate the interaction potential due to this mechanism and determine the effective $s$ wave scattering length for two fermions that interact, both directly by the interatomic potentials, as well as by the above mentioned exchange mechanism. We find that the effective scattering length is quite sensitive to changes in the condensate density and becomes strongly energy dependent. In addition, we consider the mechanical stability of these mixtures and also calculate the dispersion and the damping of the various collisionless collective modes of the gas.
\end{abstract}

\section{INTRODUCTION}

The experimental realization of Bose-Einstein condensation in trapped atomic Bose gases [1] 3] has stimulated both theoretical as well as experimental efforts to investigate new and interesting physics in ultracold atomic gases. In particular, it has spurred a lot of interest in achieving a BCS transition in trapped fermionic gases, also because a spin-polarized gas of atomic ${ }^{6} \mathrm{Li}$ has theoretically been shown to undergo a transition into a superfluid state at a critical temperature that is comparable to those realized in the experiments with trapped Bose gases [4, 栉. This relatively high critical temperature is due to it's anomalously large and negative scattering length of $-2160 \mathrm{a}_{0}$ [ [G]. To reach the temperature regime where the quantum degeneracy of the atomic gas becomes important, evaporative cooling is used. This technique, however, requires a fast thermalization rate and therefore a high collision frequency. For doubly spin-polarized Fermi gases this is not possible due to the exclusion principle, and such a high collision frequency must be obtained by using a mixture of either Fermi-Bose gases [7,8] or Fermi-Fermi gases [9]. Experimentally, this method of sympathetic cooling has already been used to produce the first two-component Bose condensate [10] and has recently also been reported to be succesful for a fermionic two-component ${ }^{40} \mathrm{~K}$ mixture [11.

Mixtures of dilute atomic gases are, however, interesting in their own right, both from an experimental as well as from a theoretical viewpoint. Indeed, experimental work on the static 
and dynamic properties of a binary mixture of Bose gases has included the study of meanfield effects [12], relative-phase coherence [13], the dynamics of component separation [14], and Rabi oscillations [15]. Furthermore, metastable states [16] and quantum tunneling effects [17] have been observed in spinor Bose-Einstein condensates, and most recently a convenient method for the creation of topological excitations in two-component Bose-condensed gases has been suggested [18] and succesfully carried out experimentally [19]. Theoretical work on trapped binary Bose condensed gases, has for example included the stability and static properties [20 22], the dynamics of the relative phase [23] and the collective modes [24]. Also for spinor Bose condensates in optical traps, the phase diagram and collective modes have been considered [25,26]. In the case of Fermi-Bose mixtures, the density profiles for gases confined in a harmonic trap have been studied at nonzero temperatures in a Thomas-Fermi approximation [27 29]. In all these cases the theory is based on a mean-field treatment of the interactions and neglects the effect of fluctuations.

Inspired by the well-known physics of ${ }^{3} \mathrm{He}^{-}{ }^{4} \mathrm{He}$ mixtures [30], we here go beyond meanfield theory and study the effective interaction between two fermions due to density fluctuations in a Bose condensate. In particular, we calculate the resulting effective interatomic $s$-wave scattering length for two fermions in different hyperfine states with the aim of manipulating it in such a way that a BCS transition becomes feasible. The two hyperfine states form an effective spin $1 / 2$ system, and therefore the mixture of fermions in two different hyperfine states can be treated as a Fermi gas with spin $1 / 2$. We mostly take the populations of the two spin levels to be equal because, if a BCS transition can be achieved at all, this will be the optimal situation. Moreover, we also consider the system to be homogeneous, for the following reason: If the mixture would be trapped in an isotropic harmonic potential with trapping frequency $\omega$, a measure for the overlap of the Bose condensate with the fermionic cloud is given by the ratio of the zero-temperature Thomas-Fermi radii for an interacting Bose condensed gas and that of an ideal Fermi gas. The former is equal to $l\left(15 N_{B} a_{B} / l\right)^{1 / 5}$ [31, where $l=\sqrt{\hbar / m \omega}$ is the harmonic oscillator length, $a_{B}$ is the bosonic scattering length, and $N_{B}$ is the number of bosons. The latter equals $l\left(48 N_{F}\right)^{1 / 6}$ [32], where $N_{F}$ is the number of fermions. For typical experimental parameters and the desirable large numbers of bosonic and fermionic atoms, this overlap of the two clouds is rather small. Therefore, in order to maximize the effect of the Bose condensate on the interaction between the fermions in the mixture, we consider only a spatially homogenous system. Although all the experiments with Bose condensed gases have been performed with harmonic oscillator traps up to now, this is not an unrealistic suggestion since it is certainly possible to create an external trapping potential that is more or less a rectangular box [33].

Physically, the effect of the Bose condensate on the fermion-fermion interaction is due to the exchange of phonons that propagate in the Bose-condensed gas. In order to calculate the effect on the interatomic interaction quantitatively, we thus, have to accurately know the density-density correlation function in the Bose gas. This is straightforward in the Bogoliubov approximation, whose validity is well-established at such low temperatures that the Bose gas is essentially fully condensed. In this manner we obtain the actual interaction potential from which we then extract the interatomic scattering length. In addition to the effective fermion-fermion interaction, we consider the important question of the stability of the three-component system against demixing of the various Bose and Fermi components of the gas. We also calculate the excitation spectrum for the Fermi-Bose mixture far below the 
Bose-Einstein transition temperature, where the dynamics of the gas is in the collisionless regime. Theoretically, this amounts to doing a so-called RPA (Random Phase Approximation) calculation for this mixture. As a result we find not only the eigenmodes of the gas, but also the Landau damping of these modes due to the imaginary part of the 'RPA-bubble' diagram. Note that from a fundamental point of view the stability and the excitation spectrum are strongly related, because a signature of the demixing-instability is the occurrence of a mode with a purely imaginary frequency.

The paper is organized as follows. In Sec. II we first calculate the effective interaction and scattering length of two fermions in the presence of a Bose condensate. In particular, we show that for realistic conditions, the scattering length strongly depends on the collision energy of the atoms, which is important when considering the prospects of a BCS transition in a spin-polarized potassium gas. In Sec. III we then consider the stability of the threecomponent system and show that in general this does not lead to very stringent constraints on the densities. Finally, in Sec. $\mathbb{I \nabla}$, we calculate the long-wavelength collective mode spectrum and damping for the Fermi-Bose mixture. We end with a summary in Sec. $\square$.

\section{EFFECTIVE INTERACTION}

In this section, we calculate the effective interaction and scattering length of two fermions. The calculation is performed by means of functional methods [34], because even in the presence of a Bose condensate, the RPA calculation for the collective modes can then be performed in a purely algebraic manner in Sec. [V] and avoids the complications of an explicit evaluation of Feynman diagrams. Moreover, it gives some more insight in the physical nature of the collisionless collective modes. Of course, it is also possible to do the same calculation in the operator formalism. If performed correctly, it gives identical results.

\section{A. Theory}

In accordance with the previous remarks, we thus start from the functional-integral expression for the grand-canonical partition function of the mixture. It reads

$$
\mathcal{Z}_{\mathrm{gr}}=\int d\left[\phi^{*}\right] d[\phi] d\left[\psi^{*}\right] d[\psi] \exp \left\{-\frac{1}{\hbar}\left(S_{B}\left[\phi^{*}, \phi\right]+S_{F}\left[\psi^{*}, \psi\right]+S_{I}\left[\phi^{*}, \phi, \psi^{*}, \psi\right]\right)\right\}
$$

and consists of an integration over a complex field $\phi(\mathbf{x}, \tau)$, which is periodic on the imaginarytime interval $[0, \hbar \beta]$, and over the Grassmann fields $\psi_{\alpha}(\mathbf{x}, \tau)$, which are antiperiodic on this interval. Therefore, $\phi(\mathbf{x}, \tau)$ describes the Bose component of the mixture, whereas $\psi_{\alpha}(\mathbf{x}, \tau)$ is associated with the Fermi components. For the latter we actually need two fields, because the fermionic atoms can be in either one of the two hyperfine states $|\alpha\rangle$. Furthermore, the total action of the mixture consists of a term for the Bose-gas

$$
S_{B}\left[\phi^{*}, \phi\right]=\int_{0}^{\hbar \beta} d \tau \int d \mathbf{x}\left\{\phi^{*}(\mathbf{x}, \tau)\left(\hbar \frac{\partial}{\partial \tau}-\frac{\hbar^{2} \nabla^{2}}{2 m_{B}}-\mu\right) \phi(\mathbf{x}, \tau)+\frac{T_{B}}{2}|\phi(\mathbf{x}, \tau)|^{4}\right\},
$$

a term for the Fermi-gas that accounts for the fact that the Pauli principle forbids $s$-wave scattering between fermionic atoms in the same hyperfine state 


$$
\begin{aligned}
S_{F}\left[\psi^{*}, \psi\right]= & \sum_{\alpha} \int_{0}^{\hbar \beta} d \tau \int d \mathbf{x}\left\{\psi_{\alpha}^{*}(\mathbf{x}, \tau)\left(\hbar \frac{\partial}{\partial \tau}-\frac{\hbar^{2} \boldsymbol{\nabla}^{2}}{2 m_{F}}-\mu_{\alpha}\right) \psi_{\alpha}(\mathbf{x}, \tau)\right. \\
& \left.+\frac{T_{F}}{2}\left|\psi_{\alpha}(\mathbf{x}, \tau)\right|^{2}\left|\psi_{-\alpha}(\mathbf{x}, \tau)\right|^{2}\right\}
\end{aligned}
$$

and a term describing the interaction between the three components of the Fermi-Bose mixture

$$
S_{I}\left[\phi^{*}, \phi, \psi^{*}, \psi\right]=\sum_{\alpha} T_{\alpha} \int_{0}^{\hbar \beta} d \tau \int d \mathbf{x}\left|\psi_{\alpha}(\mathbf{x}, \tau)\right|^{2}|\phi(\mathbf{x}, \tau)|^{2} .
$$

In these expressions we have introduced the two-body boson-boson $\mathrm{T}$ (ransition) matrix element $T_{B}=4 \pi \hbar^{2} a_{B} / m_{B}$, the two-body fermion-fermion T-matrix element $T_{F}=4 \pi \hbar^{2} a_{F} / m_{F}$, and the two two-body T-matrix elements $T_{\alpha}=2 \pi \hbar^{2} a_{\alpha} / m_{R}$ that describe the interactions between a boson and a fermion in the spinstate $|\alpha\rangle$. Here $\alpha=\{\uparrow, \downarrow\}$ denotes the hyperfine components of the Fermi-gas in the effective spin language. In addition, $\mu$ denotes the chemical potential of the Bose-gas and $\mu_{\alpha}$ denotes the chemical potentials of the two components of the Fermi gas. Note that the latter do not need to be identical, because the Fermi gas is in general not in equilibrium in spin space, due to the generally slow relaxation rates between the hyperfine degrees of freedom. Finally, the masses of the bosonic atoms and the fermionic atoms are denoted by $m_{B}$ and $m_{F}$, respectively. The reduced mass is denoted by $m_{R}=m_{F} m_{B} /\left(m_{F}+m_{B}\right)$.

Since we only consider the gas at such low temperatures that the Bose gas is essentially fully Bose condensed, we proceed by performing the usual Bogoliubov substitution for the Bose-fields, i.e., $\phi=\sqrt{n_{B}}+\phi^{\prime}$, and neglect all terms of higher than second order in $\phi^{\prime}$ or $\phi^{\prime *}$. Note that we have implicitly also neglected the depletion of the condensate and put the condensate density equal to the total density $n_{B}$ of the Bose gas. For a weakly-interacting gas with $\sqrt{n_{B} a_{B}^{3}} \ll 1$ and at low temperatures, this is clearly justified. In this manner we end up with the Bogoliubov approximation $S_{B}\left[\phi^{*}, \phi\right] \simeq \hbar \beta V T_{B} n_{B}^{2} / 2+S_{B}\left[\phi^{\prime *}, \phi^{\prime}\right]$, where $V$ is the total volume of the gas and the action for the fluctuations can be written as

$$
S_{B}\left[\phi^{\prime *}, \phi^{\prime}\right]=-\frac{\hbar}{2} \int_{0}^{\hbar \beta} d \tau d \tau^{\prime} \int d \mathbf{x} d \mathbf{x}^{\prime} \phi^{\prime \dagger}(\mathbf{x}, \tau) \cdot \mathbf{G}^{-1}\left(\mathbf{x}, \tau ; \mathbf{x}^{\prime}, \tau^{\prime}\right) \cdot \phi^{\prime}\left(\mathbf{x}^{\prime}, \tau^{\prime}\right)
$$

if we introduce the vector field

$$
\phi^{\prime}(\mathbf{x}, \tau)=\left(\begin{array}{c}
\phi^{\prime}(\mathbf{x}, \tau) \\
\phi^{\prime *}(\mathbf{x}, \tau)
\end{array}\right)
$$

and the corresponding Green's function $\mathbf{G}\left(\mathbf{x}, \tau ; \mathbf{x}^{\prime}, \tau^{\prime}\right)$ that obeys

$$
\begin{aligned}
-\hbar \mathbf{G}^{-1}\left(\mathbf{x}, \tau ; \mathbf{x}^{\prime}, \tau^{\prime}\right) & \\
& =\left(\begin{array}{cc}
\hbar \partial_{\tau}-\hbar^{2} \boldsymbol{\nabla}^{2} / 2 m+T_{B} n_{B} & T n_{B} \\
T n_{B} & -\hbar \partial_{\tau}-\hbar^{2} \nabla^{2} / 2 m+T_{B} n_{B}
\end{array}\right) \delta\left(\mathbf{x}-\mathbf{x}^{\prime}\right) \delta\left(\tau-\tau^{\prime}\right) .
\end{aligned}
$$

Note that the linear terms in $\phi^{\prime}$ and $\phi^{*}$ have dropped out of the action because of the Hugenholtz-Pines relation 


$$
\mu=T_{B} n_{B}+\sum_{\alpha} T_{\alpha} n_{\alpha}
$$

which also incorporates the mean-field effects due to the nonzero spin densities $n_{\alpha}$ in the Fermi gas. The same relation is also used to eliminate the chemical potential from Eq. (7). In addition, the interaction term in the total action becomes in the Bogoliubov approximation

$$
S_{I}\left[\phi^{\prime *}, \phi^{\prime}, \psi^{*}, \psi\right] \simeq \frac{1}{2} \int_{0}^{\hbar \beta} d \tau \int d \mathbf{x}\left\{\mathbf{J}^{\dagger}(\mathbf{x}, \tau) \cdot \phi^{\prime}(\mathbf{x}, \tau)+\phi^{\prime \dagger}(\mathbf{x}, \tau) \cdot \mathbf{J}(\mathbf{x}, \tau)\right\}
$$

with a 'current source' defined by

$$
\mathbf{J}(\mathbf{x}, \tau)=\sqrt{n_{B}} \sum_{\alpha} T_{\alpha}\left|\psi_{\alpha}(\mathbf{x}, \tau)\right|^{2}\left(\begin{array}{l}
1 \\
1
\end{array}\right) .
$$

To include the effect of phonon exchange on the interaction between two fermions, we now need to integrate over the Bose fields. This is straightforward, since it only involves the evaluation of a gaussian integral. The result for the total effective fermion action is thus

$$
\begin{aligned}
& S^{\mathrm{eff}}\left[\psi^{*}, \psi\right]=S_{F}\left[\psi^{*}, \psi\right]+\frac{1}{2 \hbar} \int_{0}^{\hbar \beta} d \tau d \tau^{\prime} \int d \mathbf{x} d \mathbf{x}^{\prime} \mathbf{J}^{\dagger}(\mathbf{x}, \tau) \cdot \mathbf{G}\left(\mathbf{x}, \tau ; \mathbf{x}^{\prime}, \tau^{\prime}\right) \cdot \mathbf{J}\left(\mathbf{x}^{\prime}, \tau^{\prime}\right) \\
& \equiv S_{F}\left[\psi^{*}, \psi\right]+\frac{1}{2} \sum_{\alpha, \alpha^{\prime}} \int_{0}^{\hbar \beta} d \tau d \tau^{\prime} \int d \mathbf{x} d \mathbf{x}^{\prime}\left|\psi_{\alpha}(\mathbf{x}, \tau)\right|^{2} V_{\alpha, \alpha^{\prime}}\left(\mathbf{x}, \tau ; \mathbf{x}^{\prime}, \tau^{\prime}\right)\left|\psi_{\alpha^{\prime}}\left(\mathbf{x}^{\prime}, \tau^{\prime}\right)\right|^{2}
\end{aligned}
$$

where $V_{\alpha, \alpha^{\prime}}\left(\mathbf{x}, \tau ; \mathbf{x}^{\prime}, \tau^{\prime}\right)$ is the effective interatomic potential due to phonon exchange. At this point, it is important to note that to be able to use in Eqs. (21), (3) and (14) the twobody T-matrix elements, instead of the real interatomic (singlet/triplet) potentials, we have already integrated out fluctuations with momenta higher than a certain cutoff $\hbar \Lambda$. Thus, in principle there is a cutoff on all the momentum integration in the rest of this paper. We will comment on the effects of this shortly. Hence, inverting Eq. (7) by means of a Fourier transformation, we find that $V_{\alpha, \alpha^{\prime}}\left(\mathbf{x}, \tau ; \mathbf{x}^{\prime}, \tau^{\prime}\right)$ is given by

$$
\begin{aligned}
& V_{\alpha, \alpha^{\prime}}\left(\mathbf{x}, \tau ; \mathbf{x}^{\prime}, \tau^{\prime}\right) \\
& \quad=-2 T_{\alpha} T_{\alpha^{\prime}} n_{B} \sum_{n} \int_{k \leq \Lambda} \frac{d \mathbf{k}}{(2 \pi)^{3}} e^{i \mathbf{k} \cdot\left(\mathbf{x}-\mathbf{x}^{\prime}\right)-i \omega_{n}\left(\tau-\tau^{\prime}\right)}\left[\frac{\epsilon(\mathbf{k})}{\left(\hbar \omega_{n}\right)^{2}+\epsilon(\mathbf{k})\left(\epsilon(\mathbf{k})+2 T_{B} n_{B}\right)}\right]
\end{aligned}
$$

where $\epsilon(\mathbf{k})=\hbar^{2} \mathbf{k}^{2} / 2 m$ and $\omega_{n}=2 \pi n / \hbar \beta$ are the even Matsubara frequencies that account for the periodicity of the Bose field $\phi(\mathbf{x}, \tau)$ and therefore of the Green's function $\mathbf{G}\left(\mathbf{x}, \tau ; \mathbf{x}^{\prime}, \tau^{\prime}\right)$. The phonon-exchange mechanism thus also induces an interaction between fermions with the same spins. Due to the Pauli-principle, however, this interaction can again at best be of a $p$-wave nature, and is in general negligible. As a result, we from now on only consider the contributions to the interaction potential between particles with opposite spin. We also neglect the frequency dependence in Eq. (12), and consider only the static contribution. This implies that the relevant collision energies of the fermions must be much less than the bosonic mean-field interaction.

The resulting instantaneous potential is given by $V\left(\mathbf{x}, \tau ; \mathbf{x}^{\prime}, \tau^{\prime}\right) \simeq V^{\mathrm{eff}}\left(\left|\mathbf{x}-\mathbf{x}^{\prime}\right|\right) \delta\left(\tau-\tau^{\prime}\right)$ with 


$$
V^{\mathrm{eff}}(r)=-\frac{\hbar^{2} m_{B} a_{\uparrow} a_{\downarrow}}{2 \pi m_{R}^{2} a_{B} \xi^{2} r} \int_{0}^{\Lambda} d k \frac{k \sin (k r)}{k^{2}+1 / \xi^{2}} .
$$

Here we have defined the coherence length $\xi=1 / \sqrt{16 \pi n_{B} a_{B}}$ in the Bose-condensed gas. The effective potential depends on three parameters, the cutoff $\Lambda$, the coherence length $\xi$ and $m_{B} a_{\uparrow} a_{\downarrow} / m_{R}^{2} a_{B}$. As we will show now, however, there is a separation of length scales, and $1 / \Lambda$ is in general much smaller than the other relevant length scale, the coherence length $\xi$. This means that we can safely ignore the cutoff and take the limit $\Lambda \rightarrow \infty$. To estimate the magnitude of the cutoff, we make use of the fact that it is determined by the requirement that the bare interatomic interaction has renormalized to the two-body scattering matrix. This renormalization of the bare interaction is described by the Lippmann-Schwinger equation

$$
\frac{1}{T_{\Lambda}}=\frac{1}{V_{0}}+\int_{\Lambda<k \leq \Lambda_{\mathrm{a}}} \frac{d \mathbf{k}}{(2 \pi)^{3}} \frac{1}{2 \epsilon(\mathbf{k})} .
$$

Here $\Lambda_{\mathrm{a}}$ denotes the ultra-violet cutoff provided by the interatomic potential, and $\Lambda$ is the momentum scale up to which fluctuations in the Bose gas are integrated over. The bare interaction $V_{0}$ is chosen such, that in the limit $\Lambda=0$ the result does not depend on the high-momentum cutoff $\Lambda_{\mathrm{a}}$ [35]. Requiring the renormalized interaction to be within approximately a fraction $x$ from the two-body transition matrix leads to

$$
\Lambda \simeq \frac{x \pi}{2 a_{B}}
$$

If we compare this value for the cutoff with the coherence length $\xi$, the product of the two is given by

$$
\xi \Lambda \simeq \frac{x \pi}{8 \sqrt{\pi n_{B} a_{B}^{3}}} .
$$

Due to the presence of the factor $\sqrt{n_{B} a_{B}^{3}}$ in the denominator, this quantity is in general much larger than 1 , and we can safely take the limit $\Lambda \rightarrow \infty$. The effective interaction in this case becomes simply

$$
V^{\mathrm{eff}}(r)=-\frac{\hbar^{2} m_{B} a_{\uparrow} a_{\downarrow}}{4 m_{R}^{2} a_{B} \xi^{2} r} e^{-r / \xi},
$$

and has the form of a purely attractive Yukawa potential.

\section{B. Results}

With this interaction potential $V^{\mathrm{eff}}(r)$, we want to determine the effective $s$-wave scattering length $a_{F}^{\text {eff }}$ for two fermions interacting also through the mechanism of phonon exchange. We can associate an $s$-wave scattering length $a_{F}^{\text {eff }}$ with the effective interaction $V^{\text {eff }}(r)$ as follows

$$
a_{F}^{\mathrm{eff}}=-\lim _{k \downarrow 0} \frac{\delta_{0}(k)}{k}
$$


Here $\delta_{0}(k)$ denotes the phase shift of the partial wave with angular momentum $l=0$. The phase shift $\delta_{0}(k)$ is defined in terms of the asymptotic form

$$
\lim _{r \rightarrow \infty} u_{0}(r ; k) \rightarrow \sin \left(k r+\delta_{0}(k)\right)
$$

for the $l=0$ partial wave $u_{0}(r ; k)$, that can be calculated from the radial Schrödinger equation

$$
\left(-\frac{d^{2}}{d r^{2}}+\frac{m_{F}}{\hbar^{2}} V^{\mathrm{eff}}(r)-k^{2}\right) u_{0}(r ; k)=0 .
$$

At this point, we have to keep in mind that there is already a scattering length $a_{F}$ for the fermions due to the interatomic potential. We can take this interatomic scattering length into account by imposing suitable boundary conditions on the partial wave $u_{0}(r ; k)$, such that we recover $a_{F}$ if the interaction due to phonon exchange vanishes, i.e., if $V^{\text {eff }}(r)=0$. The boundary conditions imposed are such that the derivative and the magnitude of the wave function at $r=0$ are equal to those of the function $\sin \left[k\left(r-a_{F}\right)\right]$. In the case of a positive scattering length, an alternative procedure would be to add a hard-core potential with a range $a_{F}$ to $V^{\text {eff }}(r)$. Both these procedures are justified because the range of the interatomic potential is much smaller than the range of the effective potential, and we have checked that numerically they indeed yield the same results.

The phase shifts due to the effective potential $V^{\text {eff }}(r)$ for mixtures of ${ }^{40} \mathrm{~K}$ and ${ }^{6} \mathrm{Li}$ with ${ }^{87} \mathrm{Rb}$, are show in Figs. 1 and 2, respectively. The interatomic scattering lengths for ${ }^{40} \mathrm{~K}-{ }^{40} \mathrm{~K}$, ${ }^{6} \mathrm{Li}^{6}{ }^{6} \mathrm{Li}$ and ${ }^{87} \mathrm{Rb}_{-}{ }^{87} \mathrm{Rb}$ collisions are taken to be $160 \mathrm{a}_{0},-2160 \mathrm{a}_{0}$ and $109 \mathrm{a}_{0}$, respectively [6. 36]. The scattering lengths for ${ }^{40} \mathrm{~K}$ and ${ }^{6} \mathrm{Li}$ with ${ }^{87} \mathrm{Rb}$ are, as far as we know, not known and we have taken them to be equal to $100 \mathrm{a}_{0}$, which is presumably a typical value. In Fig. 司 also the phase shifts for a mixture of ${ }^{40} \mathrm{~K}$ and ${ }^{39} \mathrm{~K}$ are shown, where the scattering length for ${ }^{39} \mathrm{~K}-{ }^{39} \mathrm{~K}$ and ${ }^{40} \mathrm{~K}-{ }^{39} \mathrm{~K}$ collisions has been taken to be $5 \mathrm{a}_{0}$ and $1000 \mathrm{a}_{0}$, respectively [36]. The various lines corresponds to different values of the condensate density. It is clear from these figures that for a given mixture, the phase shifts as a function of momentum asymptotically all have the same slope independent of the value of the condensate density. This slope corresponds to the interatomic scattering length $a_{F}$. However, at long wavelengths the phase shifts can be significantly different from the asymptotic limit, and can depend strongly on the collisional energy. For a mixture of ${ }^{40} \mathrm{~K}-{ }^{87} \mathrm{Rb}$ this is certainly the case. The effective interaction can even become attractive, instead of repulsive, around the Fermi momentum, which opens the possibility for a BCS transition to a superfluid phase just like the exchange of phonons in the lattice leads to superconductivity in metals [37]. For a mixture of ${ }^{6} \mathrm{Li}-$ ${ }^{87} \mathrm{Rb}$ the effects are less pronounced, and amount to a slight enhancement of the already very large and negative scattering length. The case of ${ }^{40} \mathrm{~K}-{ }^{39} \mathrm{~K}$ may be exciting, because of the possibility of a rather large scattering length for ${ }^{40} \mathrm{~K}-{ }^{39} \mathrm{~K}$ collisions, which can be of the order of $1000 \mathrm{a}_{0}$ [36]. If this value is correct, there are resonances in the effective scattering length as shown in Fig. 4 . This strongly resembles the existence of Feshbach resonances in the scattering length as a function of the applied bias magnetic field [38]. 


\section{STABILITY}

To be able to interact, the Bose and the two-component Fermi gases have to be overlapping. Therefore, we want to consider the stability of the gas against demixing of the Fermi and Bose components. To determine the stability of the mixture, we consider the matrix of second-order derivatives with respect to the densities of the free energy $F=F_{F}+F_{B}+F_{I}$, which consists of a fermion term $F_{F}$, a boson term $F_{B}$ and an interaction term $F_{I}$. For the mixture to be stable we have to require that all eigenvalues of this matrix are larger than zero, i.e., that the free-energy surface is convex. At the low temperatures of interest to us, the free-energy density is equal to the average energy density $\langle E\rangle / V$ and we get

$$
\begin{aligned}
\langle E\rangle / V & =\left\langle E_{F}\right\rangle / V+\left\langle E_{B}\right\rangle / V+\left\langle E_{I}\right\rangle / V \\
& =\frac{3}{10}\left(6 \pi^{2}\right)^{2 / 3}\left(n_{\uparrow}^{5 / 3}+n_{\downarrow}^{5 / 3}\right) \frac{\hbar^{2}}{m_{F}}+T_{F}^{\mathrm{eff}} n_{\uparrow} n_{\downarrow}+T_{B} \frac{n_{B}^{2}}{2}+\left(T_{\uparrow} n_{\uparrow}+T_{\downarrow} n_{\downarrow}\right) n_{B} .
\end{aligned}
$$

Here, the first term is the kinetic energy of the two-component Fermi gas, the second and third terms are the interaction energies of the individual Fermi and the Bose gases, respectively, and the fourth term is due to the interaction between these gases. We have neglected the kinetic energy of the Bose gas because of the low temperatures and densities. Note that the effective fermion-fermion T-matrix element $T_{F}^{\mathrm{eff}}=4 \pi \hbar^{2} a_{F}^{\text {eff }} / m_{F}$ has to be used, that includes the effect of phonon exchange. This is the case because, as long as the Fermi energy is much less than the average mean-field energy of the Bose condensate, we have to include the effect of fluctuations, which change the static properties of the mixture by renormalizing the fermion-fermion interaction as we have just discussed.

The matrix of second-order derivatives is given by

$$
\left(\begin{array}{ccc}
\frac{\partial^{2} F}{\partial n_{\uparrow} \partial n_{\uparrow}} & \frac{\partial^{2} F}{\partial n_{\uparrow} \partial n_{\downarrow}} & \frac{\partial^{2} F}{\partial n_{\uparrow} \partial n_{B}} \\
\frac{\partial^{2} F}{\partial n_{\downarrow} \partial n_{\uparrow}} & \frac{\partial^{2} F}{\partial n_{\downarrow} \partial n_{\downarrow}} & \frac{\partial^{2} F}{\partial n_{\downarrow} \partial n_{B}} \\
\frac{\partial^{2} F}{\partial n_{B} \partial n_{\uparrow}} & \frac{\partial^{2} F}{\partial n_{B} \partial n_{\downarrow}} & \frac{\partial^{2} F}{\partial n_{B} \partial n_{B}}
\end{array}\right) .
$$

Assuming that we start in the stable part of the phase diagram, the onset of an instability is signaled by the point where its determinant becomes equal to zero. Therefore, requiring the determinant of this matrix to be larger than zero is a sufficient condition for stability. It reads

$$
\begin{aligned}
4\left(\frac{\pi}{k_{\uparrow} a_{F}^{\mathrm{eff}}}\right)\left(\frac{\pi}{k_{\downarrow} a_{F}^{\mathrm{eff}}}\right) & -\frac{2}{a_{B} a_{F}^{\mathrm{eff}}} \frac{m_{B} m_{F}}{m_{R}^{2}}\left[\left(\frac{\pi}{k_{\downarrow} a_{F}^{\mathrm{eff}}}\right) a_{\uparrow}^{2}+\left(\frac{\pi}{k_{\uparrow} a_{F}^{\mathrm{eff}}}\right) a_{\downarrow}^{2}\right] \\
& +8 \frac{a_{\downarrow} a_{\uparrow}}{a_{B} a_{F}^{\mathrm{eff}}} \frac{m_{B} m_{F}}{m_{R}^{2}}-16 \geq 0,
\end{aligned}
$$

where $k_{\alpha}=\left(6 \pi^{2} n_{\alpha}\right)^{1 / 3}$ denotes the Fermi momentum associated with the spin state $|\alpha\rangle$. Note that $a_{F}^{\text {eff }}$ is a function of the condensate density $n_{B}$. In evaluating the derivatives of the free energy with respect to the density of the Bose gas, we have not taken this implicite dependence into account, which is sufficiently accurate for our purposes as long as we are not too close to a resonance. Near a resonance a more involved treatment is necessary, also because the phaseshift is then very strongly momentum dependent. 
The surface in the $n_{\alpha}-n_{B}$ volume where the equality sign holds is called the spinodal surface. The spinodal surface divides the phase space into a region where the mixture is (meta)stable, and one where it is unstable and separates into distinct phases in the stable part of the phase space. If the effect of phonon exchange is optimized by putting $n_{\uparrow}=n_{\downarrow}$, we have $k_{\downarrow}=k_{\uparrow} \equiv k_{F}$, and Eq. (22) becomes

$$
\begin{aligned}
4\left(\frac{\pi}{k_{F} a_{F}^{\text {eff }}}-2\right)\left(\frac{\pi}{k_{F} a_{F}^{\text {eff }}}+2\right) & -\frac{\left(a_{\uparrow}+a_{\downarrow}\right)^{2}}{a_{B} a_{F}^{\text {eff }}} \frac{m_{B} m_{F}}{m_{R}^{2}}\left(\frac{\pi}{k_{F} a_{F}^{\text {eff }}}-2\right) \\
& -\frac{\left(a_{\uparrow}-a_{\downarrow}\right)^{2}}{a_{B} a_{F}^{\text {eff }}} \frac{m_{B} m_{F}}{m_{R}^{2}}\left(\frac{\pi}{k_{F} a_{F}^{\text {eff }}}+2\right) \geq 0 .
\end{aligned}
$$

If we also put $a_{\uparrow}=a_{\downarrow}=a_{F B}$, the condition further simplyfies to

$$
\left(\frac{\pi}{k_{F} a_{F}^{\text {eff }}}-2\right)\left(\frac{\pi}{k_{F} a_{F}^{\text {eff }}}+2-\frac{a_{F B}^{2}}{a_{B} a_{F}^{\text {eff }}} \frac{m_{B} m_{F}}{m_{R}^{2}}\right) \geq 0 .
$$

The first factor in the left-hand side of Eq. (24) corresponds to the demixing of the two fermion components of the gas [4], whereas the second factor represents the demixing of the Bose-Einstein condensate and the fermion components [39]. Note that it depends on the various masses and scattering lengths involved, which of the two instabilities occurs first. For equal scattering lengths, i.e., $a_{F}^{\text {eff }}=a_{B}=a_{\uparrow}=a_{\downarrow} \equiv a$, the demixing of the Bose and Fermi gases always occurs first, and we reproduce the result of van Leeuwen and Cohen 40],

$$
\frac{\pi}{k_{F} a} \geq \frac{m_{B}}{m_{F}}+\frac{m_{F}}{m_{B}} .
$$

In our numerical calculations, the scattering lengths $a_{\uparrow}$ and $a_{\downarrow}$ have always been taken equal to each other and Eq. (24) applies. In the case of a mixture of ${ }^{40} \mathrm{~K}$ and ${ }^{87} \mathrm{Rb}$ this condition roughly leads for the total fermion density $n_{F}$ only to the restriction $n_{F}<10^{18}$ $\mathrm{cm}^{-3}$. For a mixture of ${ }^{6} \mathrm{Li}$ and ${ }^{87} \mathrm{Rb}$ the condition on the total density becomes $n_{F}<10^{15}$ $\mathrm{cm}^{-3}$, and for a mixture of ${ }^{40} \mathrm{~K}$ and ${ }^{39} \mathrm{~K}$ it reads $n_{F}<10^{10} \mathrm{~cm}^{-3}$. The latter condition seems to be quite restrictive. However, if we take instead of $a_{B}=5 \mathrm{a}_{0}$ a different value that is within the present uncertainty for this scattering length, i.e., $a_{B}=25 \mathrm{a}_{0}$, the condition for a mixture of ${ }^{40} \mathrm{~K}$ and ${ }^{39} \mathrm{~K}$ becomes only $n_{F}<10^{12} \mathrm{~cm}^{-3}$, which is much more favorable when one considers the prospects of achieving a BCS transition in this case. The reason is that in BCS theory, the critical temperature is given by $T_{c}=\left(8 \epsilon_{F} e^{\gamma-2} / k_{B} \pi\right) \exp \left\{-\pi \cot \left(\delta_{0}\left(k_{F}\right)\right) / 2\right\}$, with $\gamma$ Euler's constant. From this expression it follows that if the densities are low, the critical temperature for the BCS transition is also very low. Therefore, if the results of Sec. II for a ${ }^{40} \mathrm{~K}$ and ${ }^{39} \mathrm{~K}$ mixture are to be of use, as far as achieving a BCS transition is concerned, it is crucial that relatively high densities are realizable.

\section{DISPERSION OF COLLECTIVE EXCITATIONS}

We next want to consider the excitation spectrum of the gas. The collective excitations of the mixed gas are coupled modes of the fermionic spin densities, and the Bose condensate. It is therefore convenient to perform a Hubbard-Stratonovich transformation to these fermionic 
densities [41]. This amounts to introducing two real auxiliary fields $\rho_{\uparrow}(\mathbf{x}, \tau)$ and $\rho_{\downarrow}(\mathbf{x}, \tau)$, by rewriting in the integrant of the functional integral for the partition function $\mathcal{Z}_{\mathrm{gr}}$, the factor due to the fermion-fermion interaction as,

$$
\begin{aligned}
& \exp \left\{-\frac{T_{F}}{\hbar} \int_{0}^{\hbar \beta} d \tau \int d \mathbf{x}\left|\psi_{\uparrow}(\mathbf{x}, \tau)\right|^{2}\left|\psi_{\downarrow}(\mathbf{x}, \tau)\right|^{2}\right\}=\int d\left[\rho_{\uparrow}\right] d\left[\rho_{\downarrow}\right] \\
& \times \exp \left\{\frac{T_{F}}{\hbar} \int_{0}^{\hbar \beta} d \tau \int d \mathbf{x}\left[\rho_{\uparrow}(\mathbf{x}, \tau) \rho_{\downarrow}(\mathbf{x}, \tau)-\rho_{\uparrow}(\mathbf{x}, \tau)\left|\psi_{\downarrow}(\mathbf{x}, \tau)\right|^{2}-\left|\psi_{\uparrow}(\mathbf{x}, \tau)\right|^{2} \rho_{\downarrow}(\mathbf{x}, \tau)\right]\right\} .
\end{aligned}
$$

For reasons that become clear shortly, we denote the resulting action for the fermions by $S_{F}^{H}\left[\psi^{*}, \psi\right]$. It is quadratic in the fermion fields, and reads

$$
\begin{aligned}
S_{F}^{H}\left[\psi^{*}, \psi\right]=\sum_{\alpha} \int_{0}^{\hbar \beta} d \tau \int d \mathbf{x}\left\{\psi _ { \alpha } ^ { * } ( \mathbf { x } , \tau ) \left[\hbar \frac{\partial}{\partial \tau}-\frac{\hbar^{2} \boldsymbol{\nabla}^{2}}{2 m_{F}}\right.\right. & -\mu_{\alpha}+T_{F} \rho_{-\alpha}(\mathbf{x}, \tau) \\
& \left.\left.+T_{\alpha}|\phi(\mathbf{x}, \tau)|^{2}\right] \psi_{\alpha}(\mathbf{x}, \tau)\right\},
\end{aligned}
$$

Upon integrating over the fermionic fields, the grand-canonical partition function describing the gas is equal to a functional integral over the Bose field and the density fields only, with an effective action that reads

$$
\begin{aligned}
S^{\mathrm{eff}}\left[\rho_{\uparrow}, \rho_{\downarrow}, \phi^{*}, \phi\right]= & -\hbar \sum_{\alpha} \operatorname{Tr}\left\{\ln \left(-G_{\alpha}^{-1}\right)\right\}+S_{B}\left[\phi, \phi^{*}\right] \\
& -\int_{0}^{\hbar \beta} d \tau \int d \mathbf{x} T_{F} \rho_{\uparrow}(\mathbf{x}, \tau) \rho_{\downarrow}(\mathbf{x}, \tau),
\end{aligned}
$$

in terms of the Green's functions

$$
\begin{aligned}
& G_{\alpha}^{-1}\left(\mathbf{x}, \tau ; \mathbf{x}^{\prime}, \tau^{\prime}\right) \\
& \quad=-\frac{1}{\hbar}\left\{\hbar \frac{\partial}{\partial \tau}-\frac{\hbar^{2} \boldsymbol{\nabla}^{2}}{2 m}-\mu_{\alpha}+T_{F} \rho_{-\alpha}(\mathbf{x}, \tau)+T_{\alpha}|\phi(\mathbf{x}, \tau)|^{2}\right\} \delta\left(\mathbf{x}-\mathbf{x}^{\prime}\right) \delta\left(\tau-\tau^{\prime}\right) .
\end{aligned}
$$

Expanding this action around its minimum, by requiring the linear terms in the fluctuations to be zero, will result in the Hartree approximation for the equilibrium densities. This is sufficiently accurate because we are dealing with a fermionic gas in a nonmagnetic phase, where the Fock term in the selfenergy is zero, due to the spin-symmetry of the action. It also explains the use of the symbol H(artree) in Eq. (27). Inserting thus into the right-hand side of Eq. (28)

$$
\rho_{\alpha}(\mathbf{x}, \tau)=n_{\alpha}+\rho_{\alpha}^{\prime}(\mathbf{x}, \tau)
$$

and

$$
\phi(\mathbf{x}, \tau)=\sqrt{n_{B}}+\phi^{\prime}(\mathbf{x}, \tau)
$$

we obtain from the requirement that the linear terms in the fluctuations vanish again the Hugenholtz-Pines relation 


$$
-\mu+T_{B} n_{B}+\sum_{\alpha} T_{\alpha} G_{\alpha}^{H}(\mathbf{x}, \tau ; \mathbf{x}, \tau)=0
$$

and in addition the expected equation for the average spin densities

$$
n_{\alpha}=G_{\alpha}^{H}(\mathbf{x}, \tau ; \mathbf{x}, \tau)
$$

where

$$
G_{\alpha}^{H^{-1}}\left(\mathbf{x}, \tau ; \mathbf{x}^{\prime}, \tau^{\prime}\right)=-\frac{1}{\hbar}\left\{\hbar \frac{\partial}{\partial \tau}-\frac{\hbar^{2} \nabla^{2}}{2 m}-\mu_{\alpha}+T_{F} \rho_{-\alpha}+T_{\alpha} n_{B}\right\} \delta\left(\mathbf{x}-\mathbf{x}^{\prime}\right) \delta\left(\tau-\tau^{\prime}\right)
$$

is the usual fermionic one-particle propagator, or two-point correlation function, in the Hartree approximation. We, therefore, recognise in Eqs. (32), (33), and (34) the selfconsistent Hartree equations for the boson and fermion densities at given chemical potentials $\mu$, $\mu_{\uparrow}$, and $\mu_{\downarrow}$.

To find the theory describing the fluctuations around this equilibrium, we have to perform the expansion around this minimum up to second order in the fluctuations $\rho_{\alpha}^{\prime}$ and $\phi^{\prime}$. The poles in the Green's function of the resulting theory give us the desired dispersion of the collective modes, for they are also the poles in the linear response of the densities to an external perturbation. If we introduce again the vector notation

$$
\phi_{\mathbf{k}, n}^{\prime}=\left(\begin{array}{c}
\phi_{\mathbf{k}, n}^{\prime} \\
\phi_{-\mathbf{k},-n}^{\prime *}
\end{array}\right)
$$

and also

$$
\rho_{\mathbf{k}, n}^{\prime}=\left(\begin{array}{c}
\rho_{\uparrow ; \mathbf{k}, n}^{\prime} \\
\rho_{\downarrow ; \mathbf{k}, n}^{\prime}
\end{array}\right)
$$

the quadratic part of the effective action for the fluctuations $\boldsymbol{\rho}_{\alpha}^{\prime}$ and $\boldsymbol{\phi}^{\prime}$ can be conveniently written in momentum space as

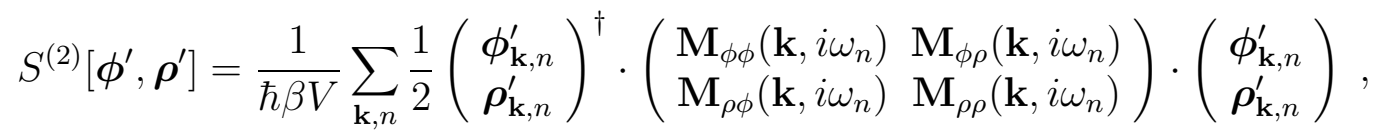

where we have defined the matrices

$$
\mathbf{M}_{\phi \phi}\left(\mathbf{k}, i \omega_{n}\right)=\left(\begin{array}{cc}
-i \hbar \omega_{n}+\epsilon(\mathbf{k})+\Sigma\left(\mathbf{k}, i \omega_{n}\right) & \Sigma\left(\mathbf{k}, i \omega_{n}\right) \\
\Sigma\left(\mathbf{k}, i \omega_{n}\right) & i \hbar \omega_{n}+\epsilon(\mathbf{k})+\Sigma\left(\mathbf{k}, i \omega_{n}\right)
\end{array}\right),
$$

and

$$
\mathbf{M}_{\rho \rho}\left(\mathbf{k}, i \omega_{n}\right)=T_{F}\left(\begin{array}{cc}
T_{F} \Pi_{\downarrow}\left(\mathbf{k}, i \omega_{n}\right) & -1 \\
-1 & T_{F} \Pi_{\uparrow}\left(\mathbf{k}, i \omega_{n}\right)
\end{array}\right)
$$

and

$$
\mathbf{M}_{\rho \phi}^{T}\left(\mathbf{k}, i \omega_{n}\right)=\mathbf{M}_{\phi \rho}\left(\mathbf{k}, i \omega_{n}\right)=T_{F} \sqrt{n_{B}}\left(\begin{array}{cc}
T_{\downarrow} \Pi_{\downarrow}\left(\mathbf{k}, i \omega_{n}\right) & T_{\uparrow} \Pi_{\uparrow}\left(\mathbf{k}, i \omega_{n}\right) \\
T_{\downarrow} \Pi_{\downarrow}\left(\mathbf{k}, i \omega_{n}\right) & T_{\uparrow} \Pi_{\uparrow}\left(\mathbf{k}, i \omega_{n}\right)
\end{array}\right) .
$$


We have also introduced the selfenergy of the Bose gas

$$
\Sigma\left(\mathbf{k}, i \omega_{n}\right)=T_{B} n_{B}+\left[T_{\uparrow}^{2} \Pi_{\uparrow}\left(\mathbf{k}, i \omega_{n}\right)+T_{\downarrow}^{2} \Pi_{\downarrow}\left(\mathbf{k}, i \omega_{n}\right)\right] n_{B}
$$

and the RPA-bubble, or equivalently, the density-density response function of the ideal Fermi gas

$$
\Pi_{\alpha}\left(\mathbf{k}, i \omega_{n}\right)=\int \frac{d \mathbf{p}}{(2 \pi)^{3}} \frac{n\left(\epsilon_{\alpha}(\mathbf{k}+\mathbf{p})\right)-n\left(\epsilon_{\alpha}(\mathbf{p})\right)}{-i \hbar \omega_{n}+\epsilon_{\alpha}(\mathbf{k}+\mathbf{p})-\epsilon_{\alpha}(\mathbf{p})}
$$

where the dispersions obey $\epsilon_{\alpha}(\mathbf{k})=\hbar^{2} k^{2} / 2 m+T_{F} \rho_{-\alpha}+T_{\alpha} n_{B}-\mu_{\alpha}$, and $n(\epsilon)=1 /(\exp (\beta \epsilon)+1)$ is the usual Fermi-Dirac distribution function. Note that $\Sigma\left(\mathbf{k}, i \omega_{n}\right)$ and $\Pi_{\alpha}\left(\mathbf{k}, i \omega_{n}\right)$ are invariant under the substitution $\left(\mathbf{k}, i \omega_{n}\right) \rightarrow\left(-\mathbf{k},-i \omega_{n}\right)$, due to the time-reversal symmetry of the problem.

In the long-wavelength limit, i.e., for small $k$, Eq. (42) can be rewritten as

$$
\Pi_{\alpha}\left(\mathbf{k}, i \omega_{n}\right)=\frac{m k_{\alpha}}{2 \hbar^{2} \pi^{2}} \int_{0}^{\infty} d \epsilon \frac{\partial n_{\alpha}}{\partial \epsilon} \sqrt{\epsilon}\left[1-\frac{x_{\alpha, n}}{\sqrt{\epsilon}} \arctan \frac{\sqrt{\epsilon}}{x_{\alpha, n}}\right],
$$

where $x_{\alpha, n}=m \omega_{n} / \hbar k_{\alpha} k$,

$$
n_{\alpha}(\epsilon)=\left[e^{\beta\left(\epsilon_{\alpha} \epsilon-\mu_{\alpha}+T_{F} \rho_{-\alpha}+T_{\alpha} n_{B}\right)}+1\right]^{-1},
$$

and we have defined the Fermi energies and wavevectors by $\epsilon_{\alpha}=\mu_{\alpha}-T_{F} \rho_{-\alpha}-T_{\alpha} n_{B} \equiv$ $\hbar^{2} k_{\alpha}^{2} / 2 m$. The analytic continuation of Eq. (43) to physical energies $\omega=i \omega_{n}$ reads

$$
\Pi_{\alpha}(\mathbf{k}, \omega)=\frac{m k_{\alpha}}{2 \hbar^{2} \pi^{2}} \int_{0}^{\infty} d \epsilon \frac{\partial n_{\alpha}}{\partial \epsilon}\left[1-\frac{x_{\alpha}}{2} \log \left|\frac{x_{\alpha}+\sqrt{\epsilon}}{x_{\alpha}-\sqrt{\epsilon}}\right|-i \frac{\pi}{2}\left|x_{\alpha}\right| \theta\left(\sqrt{\epsilon}-\left|x_{\alpha}\right|\right)\right],
$$

with $x_{\alpha}=m \omega / \hbar k_{\alpha} k$. We want to find the zero-temperature result and the lowest-order corrections in the temperature. This can be done by means of a Sommerfeld expansion, which amounts to expanding the real part of the expression between square brackets in Eq. (45) around $\epsilon=1$. Doing so, we find in first instance

$$
\begin{aligned}
\Pi_{\alpha}(\mathbf{k}, \omega)= & \frac{m k_{\alpha}}{2 \hbar^{2} \pi^{2}} \int_{0}^{\infty} d \epsilon \frac{\partial n_{\alpha}}{\partial \epsilon}\left\{1-\frac{x_{\alpha}}{2} \log \left|\frac{x_{\alpha}+1}{x_{\alpha}-1}\right|\right. \\
& -\left[1-\frac{x_{\alpha}^{2}\left(x_{\alpha}^{2}-3\right)}{\left(1-x_{\alpha}^{2}\right)^{2}}\right] \frac{(\epsilon-1)^{2}}{8}+\mathcal{O}\left[(\epsilon-1)^{4}\right] \\
& \left.-i \frac{\pi}{2}\left|x_{\alpha}\right| \theta\left(\sqrt{\epsilon}-\left|x_{\alpha}\right|\right)\right\} .
\end{aligned}
$$

Integrating then over $\epsilon$, we obtain for the real part in lowest order

$$
\Pi_{\alpha}^{(0)}(\mathbf{k}, \omega)=-\frac{m k_{\alpha} n_{\alpha}(0)}{2 \hbar^{2} \pi^{2}}\left[1-\frac{x_{\alpha}}{2} \log \left|\frac{x_{\alpha}+1}{x_{\alpha}-1}\right|\right],
$$

and for the imaginary part exactly

$$
\operatorname{Im}\left[\Pi_{\alpha}(\mathbf{k}, \omega)\right]=\frac{m k_{\alpha} n_{\alpha}(0)}{2 \hbar^{2} \pi^{2}} \frac{\pi}{2 n_{\alpha}(0)}\left|x_{\alpha}\right| n_{\alpha}\left(\left|x_{\alpha}\right|^{2}\right) .
$$


At zero temperature Eqs. (47) and (48) reduce to the well-known result for the zerotemperature RPA bubble 42. Note that the imaginairy part in Eq. (48) is valid for all temperatures and cannot simply be expanded as a power series in $k_{B} T / \epsilon_{F}$. This is not true for the real part of $\Pi_{\alpha}(\mathbf{k}, \omega)$ and the lowest-order temperature correction is given by

$$
\Pi_{\alpha}^{(2)}(\mathbf{k}, \omega)=-\frac{m k_{\alpha}}{16 \hbar^{2} \pi^{2}}\left[1-\frac{x_{\alpha}^{2}\left(x_{\alpha}^{2}-3\right)}{\left(1-x_{\alpha}^{2}\right)^{2}}\right]\left(\frac{k_{B} T}{\epsilon_{\alpha}}\right)^{2} \frac{\pi^{2}}{3} .
$$

To find the long-wavelength dispersion, i.e., $\omega=c k$ or equivalently $x_{\alpha}=m c / \hbar k_{\alpha}$, of the collective modes of the mixture, we require that the determinant of the fluctuation matrix in Eq. (37) equals zero. Calculating the determinant, the terms of $\mathcal{O}\left(k^{0}\right)$ drop out, indicating that the collective excitations are indeed gapless as assumed by our ansatz $\omega=c k$. Leaving out terms that are of $\mathcal{O}\left(k^{4}\right)$, because they do not affect the linear part of the dispersion relation, and dividing out an overall factor of $k^{2}$, we ultimately find the result

$$
\begin{aligned}
& {\left[T_{F}^{2} \Pi_{\uparrow}(c) \Pi_{\downarrow}(c)-1\right]\left[-m c^{2}+\Sigma(c)\right] } \\
- & T_{F} n_{B}\left[T_{F} \Pi_{\uparrow}(c) T_{\downarrow} \Pi_{\downarrow}(c)-T_{\uparrow} \Pi_{\uparrow}(c)\right] T_{\downarrow} \Pi_{\downarrow}(c) \\
- & T_{F} n_{B}\left[T_{F} \Pi_{\downarrow}(c) T_{\uparrow} \Pi_{\uparrow}(c)-T_{\downarrow} \Pi_{\downarrow}(c)\right] T_{\uparrow} \Pi_{\uparrow}(c)=0 .
\end{aligned}
$$

Here, we have for convenience introduced the shorthand notation $\Pi_{\alpha}(c)=\Pi_{\alpha}(\mathbf{k}, c k)$. If we put $T_{\uparrow}=T_{\downarrow}=0$, this simply gives

$$
\left[T_{F}^{2} \Pi_{\uparrow}(c) \Pi_{\downarrow}(c)-1\right]\left[-m c^{2}+\Sigma(c)\right]=0 .
$$

The first factor describes the collective modes of the Fermi gas and the second factor describes the Bogoliubov modes of the condesate, which are of course decoupled in this case. For small values of $T_{\uparrow}$ and $T_{\downarrow}$ these modes also exist, but the dispersion is changed. The lowest-order correction in the boson-fermion scattering lengths $a_{\alpha}$ to the Bogoliubov speed of sound $c_{0}=\sqrt{T_{B} n_{B} / m}$ is determined by

$$
\begin{aligned}
m c_{1}^{2}=\Sigma\left(c_{0}\right) & -\frac{T_{F} n_{B}\left[T_{F} \Pi_{\uparrow}\left(c_{0}\right) T_{\downarrow} \Pi_{\downarrow}\left(c_{0}\right)-T_{\uparrow} \Pi_{\uparrow}\left(c_{0}\right)\right] T_{\downarrow} \Pi_{\downarrow}\left(c_{0}\right)}{\left[T_{F}^{2} \Pi_{\uparrow}\left(c_{0}\right) \Pi_{\downarrow}\left(c_{0}\right)-1\right]} \\
& -\frac{T_{F} n_{B}\left[T_{F} \Pi_{\downarrow}\left(c_{0}\right) T_{\uparrow} \Pi_{\uparrow}\left(c_{0}\right)-T_{\downarrow} \Pi_{\downarrow}\left(c_{0}\right)\right] T_{\uparrow} \Pi_{\uparrow}\left(c_{0}\right)}{\left[T_{F}^{2} \Pi_{\uparrow}\left(c_{0}\right) \Pi_{\downarrow}\left(c_{0}\right)-1\right]} .
\end{aligned}
$$

To find the full solution we can just iterate Eq. (52) and the result converges rapidly to a solution of Eq. (50). Note that Eq. (52) has an imaginary part and therefore also describes the damping of the Bogoliubov mode. Under the circumstances that we have studied in Secs. II and III the correction on the speed of sound due to the presence of the Fermi gas is small and at most about $5 \%$ of the uncoupled Bogoliubov result. This is important for our purposes, because it shows that for the calculation of the effective fermion-fermion interaction, we do not need to selfconsistently include the effect of the Fermi gas on the density fluctuations of the condensate.

To find the other propagating solutions of Eq. (50) we need to be more careful. For clarity, we first again treat the case where $T_{\uparrow}=T_{\downarrow}=0$. The zero-sound modes are now the solutions of 


$$
\left[T_{F}^{2} \Pi_{\uparrow}(c) \Pi_{\downarrow}(c)-1\right]=0 .
$$

The product $\Pi_{\uparrow}(c) \Pi_{\downarrow}(c)$ diverges logarithmically for two value of $c$, i.e., for the Fermi velocities $c_{\alpha}=\hbar k_{\alpha} / m$. In principle there are therefore four solutions to this equation, which can be found by expanding around either $c_{\uparrow}$ or $c_{\downarrow}$. Without loss of generality, we assume that $c_{\uparrow}>c_{\downarrow}$. To find then the solution that is not overdamped, we have to expand around $c_{\uparrow}$ and find the solution which is slightly bigger than $c_{\uparrow}$, i.e., $c=c_{\uparrow}+\delta c$ with $\delta c>0$. In this way we make sure that the imaginary parts of both $\Pi_{\uparrow}(c)$ and $\Pi_{\downarrow}(c)$ are equal to zero at zero temperature. Experimentally we are always in the weak-coupling limit, which implies that $T_{F} \ll \hbar^{2} / m k_{\uparrow}$. In this case the zero-temperature expressions for $\Pi_{\uparrow}(c)$ and $\Pi_{\downarrow}(c)$ become explicitly

$$
\Pi_{\uparrow}^{0}\left(c_{\uparrow}+\delta c\right)=-\frac{m k_{\uparrow}}{2 \hbar^{2} \pi^{2}}\left[1-\frac{1}{2} \log \frac{2 c_{\uparrow}}{\delta c}\right]+\mathcal{O}(\delta c)
$$

and

$$
\Pi_{\downarrow}^{0}\left(c_{\uparrow}+\delta c\right)=-\frac{m k_{\downarrow}}{2 \hbar^{2} \pi^{2}}\left[1-\frac{1}{2}\left(\frac{c_{\uparrow}}{c_{\downarrow}}\right) \log \frac{\left(\frac{c_{\uparrow}}{c_{\downarrow}}\right)+1}{\left(\frac{c_{\uparrow}}{c_{\downarrow}}\right)+\frac{\delta c}{c_{\downarrow}}-1}\right]+\mathcal{O}(\delta c),
$$

respectively. In the limit that $\left(c_{\uparrow}-c_{\downarrow}\right) \ll \delta c$ we thus get

$$
\delta c=2 c_{\uparrow} \exp \left(-\frac{4 \pi^{2} \hbar^{2}}{m k_{\uparrow} T_{F}}-2\right),
$$

where we have made use of the fact that in this limit $k_{\downarrow}-k_{\uparrow}=\mathcal{O}(\delta c)$. Note that our result differs from that of Fetter and Walecka [42]. They use an approximation that does not obey the Pauli exclusion principle, because there is s-wave scattering between particles that are in the same spin-state. As a result, their mean-field energy for an atom in the spin state $|\alpha\rangle$ is $T_{F} \sum_{\alpha} n_{\alpha}$ instead of $T_{F} n_{-\alpha}$. For $n_{\uparrow}=n_{\downarrow}$ this effectively means a factor of two reduction of the interaction strength. In the limit that $\left(c_{\uparrow}-c_{\downarrow}\right) \gg \delta c$ we find at zero temperature

$$
\delta c=2 c_{\uparrow} \exp \left(-\frac{4 \pi^{2} \hbar^{2}}{m k_{\uparrow} T_{F}^{2} \Pi_{\downarrow}^{0}\left(c_{\uparrow}\right)}-2\right) .
$$

Let us now consider the effect of the presence of the Bose condensate and do the same calculation for the mixture of the Bose condensed gas with the two-component fermion gas. In the limit that $\left(c_{\uparrow}-c_{\downarrow}\right) \ll \delta c$ we again find

$$
\delta c=2 c_{\uparrow} \exp \left(-\frac{4 \pi^{2} \hbar^{2}}{m k_{\uparrow} T_{F}}-2\right) .
$$

This is the same result as in Eq. (56) where the fermionic and the Bose condensed gas are decoupled, and is due to the fact that in this limit the zero-sound mode is a pure spin wave. The density profile is therefore constant and, since the fermionic and the Bose condensed gas couple only through the density fluctuations, there is no effect of the presence of the Bose gas. On the other hand, in the limit that $\left(c_{\uparrow}-c_{\downarrow}\right) \gg \delta c$ we now obtain 


$$
\delta c=2 c_{\uparrow} \exp \left(-\frac{4 \pi^{2} \hbar^{2}}{m k_{\uparrow} T_{F} \chi}-2\right),
$$

where we have defined the quantity

$$
\chi=\frac{T_{F}\left(-m c_{\uparrow}^{2}+T_{B} n_{B}+T_{\downarrow}^{2} \Pi_{\downarrow}^{0}\left(c_{\uparrow}\right) n_{B}\right)}{T_{F}^{2} \Pi_{\downarrow}^{0}\left(c_{\uparrow}\right)\left(-m c_{\uparrow}^{2}+T_{B} n_{B}\right)-T_{\uparrow}^{2} n_{B}+2 T_{F} T_{\downarrow} T_{\uparrow} \Pi_{\downarrow}^{0}\left(c_{\uparrow}\right) n_{B}} .
$$

Hence, Eq. (59) reduces to Eq. (57) if we take $T_{\uparrow}=T_{\downarrow}=0$, as it should. The same is of course true if $n_{B}=0$. Note that Eq. (49) offers the opportunity to obtain also the small nonzero-temperature corrections to the above results, if this turns out to be necessary for a particular application.

\section{CONCLUSIONS}

We have calculated the effect of phonon exchange on the scattering length for two fermions with different spins. Under the right circumstances this effect can be quite large and is then a possible way to experimentally tune the fermion-fermion scattering length. This may in particular be useful for the achievement of a BCS transition in a mixture of ${ }^{40} \mathrm{~K}$ and ${ }^{39} \mathrm{~K}$, although it appears that a more precise determination of the various scattering lengths involved in this case is necessary to make sure of this. In addition, we have analyzed the stability and the mode structure of the Bose-Fermi mixture. Our results in the latter case, which are valid for an arbitrary ratio of the densities of the three components, agree in limiting cases with expressions obtained by other authors 43.

\section{ACKNOWLEDGMENTS}

We would like to thank C.J. Pethick and E. Braaten for usefull comments. 


\section{REFERENCES}

[1] M.H. Anderson, J.R. Ensher, M.R. Matthews, C.E. Wieman and, E.A. Cornell, Science 269, 198 (1995).

[2] C.C. Bradley, C.A. Sackett, J.J. Tollett and, R.G. Hulet, Phys. Rev. Lett. 75, 1687 (1995); C.C. Bradley, C.A. Sackett and, R.G. Hulet, ibid. 78, 985 (1997).

[3] K.B. Davis, M.O. Mewes, M.R. Andrews, N.J. van Druten, D.S. Durfee, D.M. Kurn and, W. Ketterle, Phys. Rev. Lett. 75, 3969 (1995).

[4] M. Houbiers, R. Ferwerda, H.T.C. Stoof, W.I. McAlexander, C.A. Sackett, and R.G. Hulet, Phys. Rev. A 56, 4864 (1997).

[5] See also A.G.K. Modawi and A.J. Legget, J. Low. Temp. Phys. 109, 625 (1997); M.A. Baranov and D.S. Petrov, Phys. Rev. A58, R801 (1998); G. Bruun, Y. Castin, R. Dum, and K. Burnett, Eur. Phys. J. D 7, 433 (1999).

[6] E.R.I. Abraham, W.I. McAlexander, J.M. Gerton, and R.G. Hulet, Phys. Rev. A 55, R3299 (1997).

[7] E. Timmermans and R. Côté, Phys. Rev. Lett. 80, 3419 (1998).

[8] W. Geist, L. You, and T.A.B. Kennedy, Phys. Rev. A 59, 1500 (1999).

[9] H.T.C. Stoof, M. Houbiers, C.A. Sackett, and R.G. Hulet, Phys. Rev. Lett. 76, 10 (1996).

[10] C.J. Myatt, A.E. Burt, R.W. Ghrist, E.A. Cornell, and C.E. Wieman, Phys. Rev. Lett. 78, 586 (1997).

[11] B. DeMarco and D.S. Jin, Science 285, 1703 (1999).

[12] M.R. Matthews, D.S. Hall, D.S. Jin, J.R. Ensher, C.E. Wieman, E.A. Cornell, F. Dalfovo, C. Minniti, and S. Stringari, Phys. Rev. Lett. 81, 243 (1998).

[13] D.S. Hall, M.R. Matthews, J.R. Ensher, C.E. Wieman, and E.A. Cornell, Phys. Rev. Lett. 81, 1539 (1998).

[14] D.S. Hall, M.R. Matthews, C.E. Wieman, and E.A. Cornell, Phys. Rev. Lett. 81, 1543 (1998).

[15] M.R. Matthews, B.P. Anderson, P.C. Haljan, D.S. Hall, M.J. Holland, J.E. Williams, C.E. Wieman, and E.A. Cornell, Phys. Rev. Lett. 83, 3358 (1999).

[16] H.-J. Miesner, D.M. Stamper-Kurn, J. Stenger, S. Inouye, A.P. Chikkatur, and W. Ketterle, Phys. Rev. Lett. 82, 2228 (1999).

[17] D.M. Stamper-Kurn, H.-J. Miesner, A.P. Chikkatur, S. Inouye, J. Stenger, and W. Ketterle, Phys. Rev. Lett. 83, 661 (1999).

[18] J.E. Williams and M.J. Holland, Nature 401, 568 (1999).

[19] M.R. Matthews, B.P. Anderson, P.C. Haljan, D.S. Hall, C.E. Wieman, and E.A. Cornell, Phys. Rev. Lett. 83, 2498 (1999).

[20] T.-L. Ho and V.B. Shenoy, Phys. Rev. Lett. 77, 3276 (1996).

[21] B.D. Esry, C.H. Greene, J.P. Burke, Jr., and J.L. Bohn, Phys. Rev. Lett. 78, 3594 (1997).

[22] P. Öhberg and S. Stenholm, Phys. Rev. A, 57, 1272 (1998); ibid 59, 3890 (1999).

[23] A. Sinatra and Y. Castin, (unpublished, cond-mat/9904353)

[24] Th. Busch, J. I. Cirac, V.M. Pérez-Garcia, and P. Zoller, Phys. Rev. A 562978 (1997).

[25] T.-L. Ho, Phys. Rev. Lett. 81, 742 (1998).

[26] C.K. Law, H. Pu, and N.P. Bigelow, Phys. Rev. Lett. 81, 5257 (1998).

[27] K. Mølmer, Phys. Rev. Lett. 80, 1804 (1998). 
[28] L. Vichi, M. Inguscio, S. Stringari and G.M. Tino, J. Phys. B 31, L899 (1998).

[29] M. Amoruso, A. Minguzzi, S. Stringari, M.P. Tosi, and L. Vichi, Eur. Phys. J. D 4, 261 (1998).

[30] See, for instance, J. Bardeen, G. Baym, and D. Pines, Phys. Rev. Lett. 17, 372 (1966); Phys. Rev. 156, 207 (1967).

[31] G. Baym and C.J. Pethick, Phys. Rev. Lett. 76, 6 (1996).

[32] D.A. Butts and D.S. Rokhsar, Phys. Rev. A 55, 4346 (1997).

[33] R.G. Hulet and E.A. Cornell, private communications.

[34] For a recent introduction see, H.T.C. Stoof (unpublished, cond-mat/9910441).

[35] M. Bijlsma and H.T.C. Stoof, Phys. Rev. A 54, 5085 (1996).

[36] B. DeMarco, J.L. Bohn, J.P. Burke Jr., M.J. Holland, and D.S. Jin, Phys. Rev. Lett. 82, 4208 (1999); J.L. Bohn, J.P. Burke, Jr., C.H. Greene, H. Wang, P.L. Gould and W.C. Stwalley, Phys. Rev. A 59, 3660 (1999).

[37] J. Bardeen, L.N. Cooper, and J.R. Schrieffer, Phys. Rev. 108, 1175 (1957).

[38] E. Tiesinga, B.J. Verhaar, and H.T.C. Stoof, Phys. Rev. A 47, 4114 (1993). For a discussion of Feshbach resonances in 40K see, J.L. Bohn (unpublished, cond-mat/9911132).

[39] The case of a single fermionic component is considered by L. Viverit, C.J. Pethick, and H. Smith (unpublished, cond-mat/9911080).

[40] J.M.J. van Leeuwen and E.D.G. Cohen, Phys. Rev. 176, 385, (1968).

[41] H. Kleinert, Fort. Phys. 26, 565 (1978).

[42] A.L. Fetter and J.D. Walecka, Quantum theory of many-particle systems (McGraw-Hill, New York, 1971).

[43] S.-K. Yip and T.-L. Ho, Phys. Rev. A 59, 4653 (1999). 


\section{FIGURES}

FIG. 1. The phase shift $\delta_{0}$ as a function of the momentum $k$, for a mixture of ${ }^{40} \mathrm{~K}$ and ${ }^{87} \mathrm{Rb}$. The condensate density is (1) $n_{B}=1 \times 10^{10} \mathrm{~cm}^{-3}$, (2) $n_{B}=1 \times 10^{13} \mathrm{~cm}^{-3}$, and (3) $n_{B}=1 \times 10^{14}$ $\mathrm{cm}^{-3}$.

FIG. 2. The phase shift $\delta_{0}$ as a function of the momentum $k$, for a mixture of ${ }^{6} \mathrm{Li}$ and ${ }^{87} \mathrm{Rb}$. The condensate density is $(1) n_{B}=1 \times 10^{10} \mathrm{~cm}^{-3}$, (2) $n_{B}=1 \times 10^{13} \mathrm{~cm}^{-3}$, and (3) $n_{B}=1 \times 10^{14}$ $\mathrm{cm}^{-3}$.

FIG. 3. The phase shift $\delta_{0}$ as a function of the momentum $k$, for a mixture of ${ }^{40} \mathrm{~K}$ and ${ }^{39} \mathrm{~K}$. The condensate density is (1) $n_{B}=4 \times 10^{12} \mathrm{~cm}^{-3}$, (2) $n_{B}=5 \times 10^{12} \mathrm{~cm}^{-3}$, (3) $n_{B}=6 \times 10^{12}$ $\mathrm{cm}^{-3}$, (4) $n_{B}=7 \times 10^{12} \mathrm{~cm}^{-3}$, (5) $n_{B}=8 \times 10^{12} \mathrm{~cm}^{-3}$.

FIG. 4. The scattering length $a_{F}^{\text {eff }}$ as a function of the density of condensed atoms $n_{B}$, for a mixture of ${ }^{40} \mathrm{~K}$ and ${ }^{39} \mathrm{~K}$. 
M.J.Bijlsma et al. fig.1

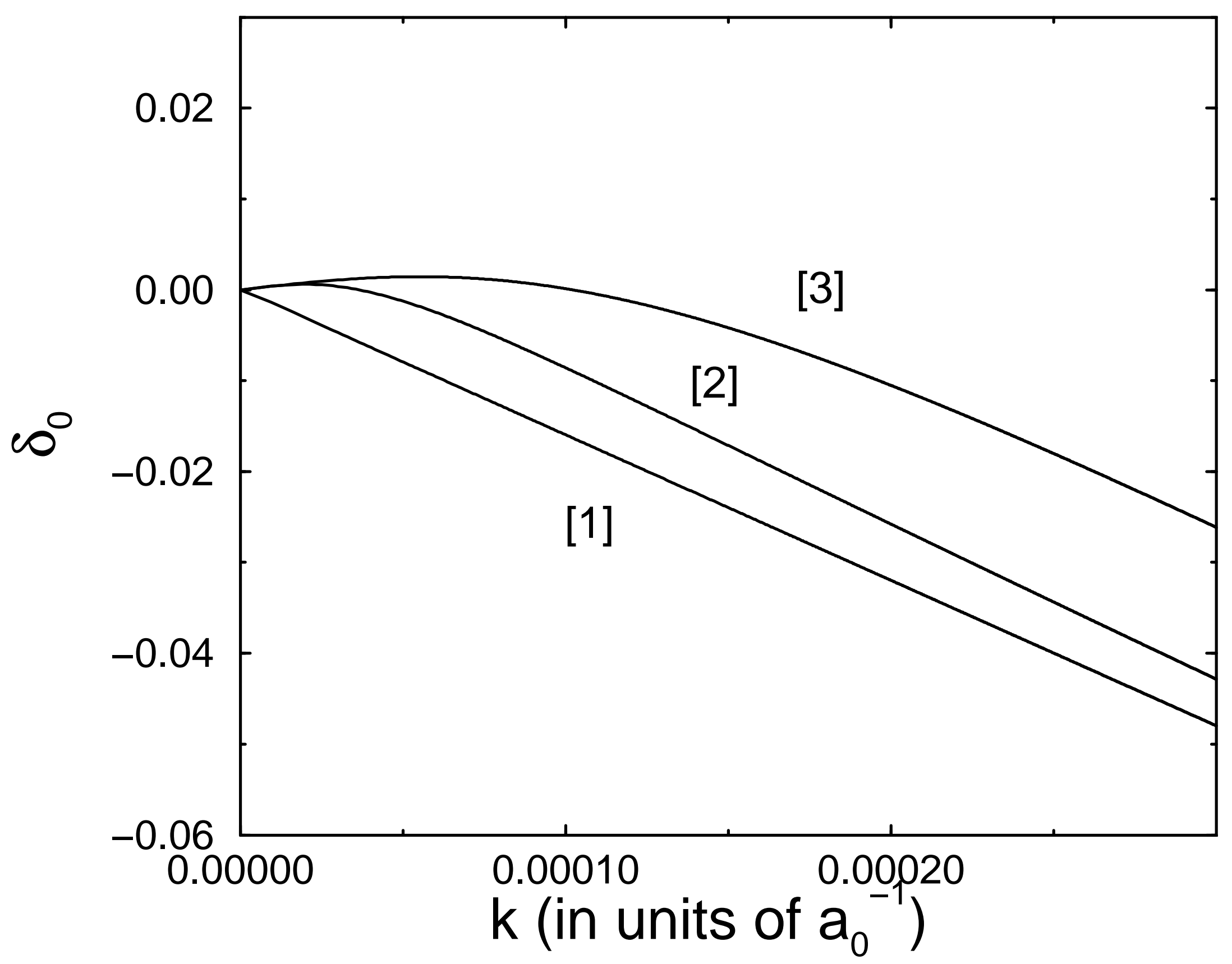


M.J.Bijlsma et al. fig.2

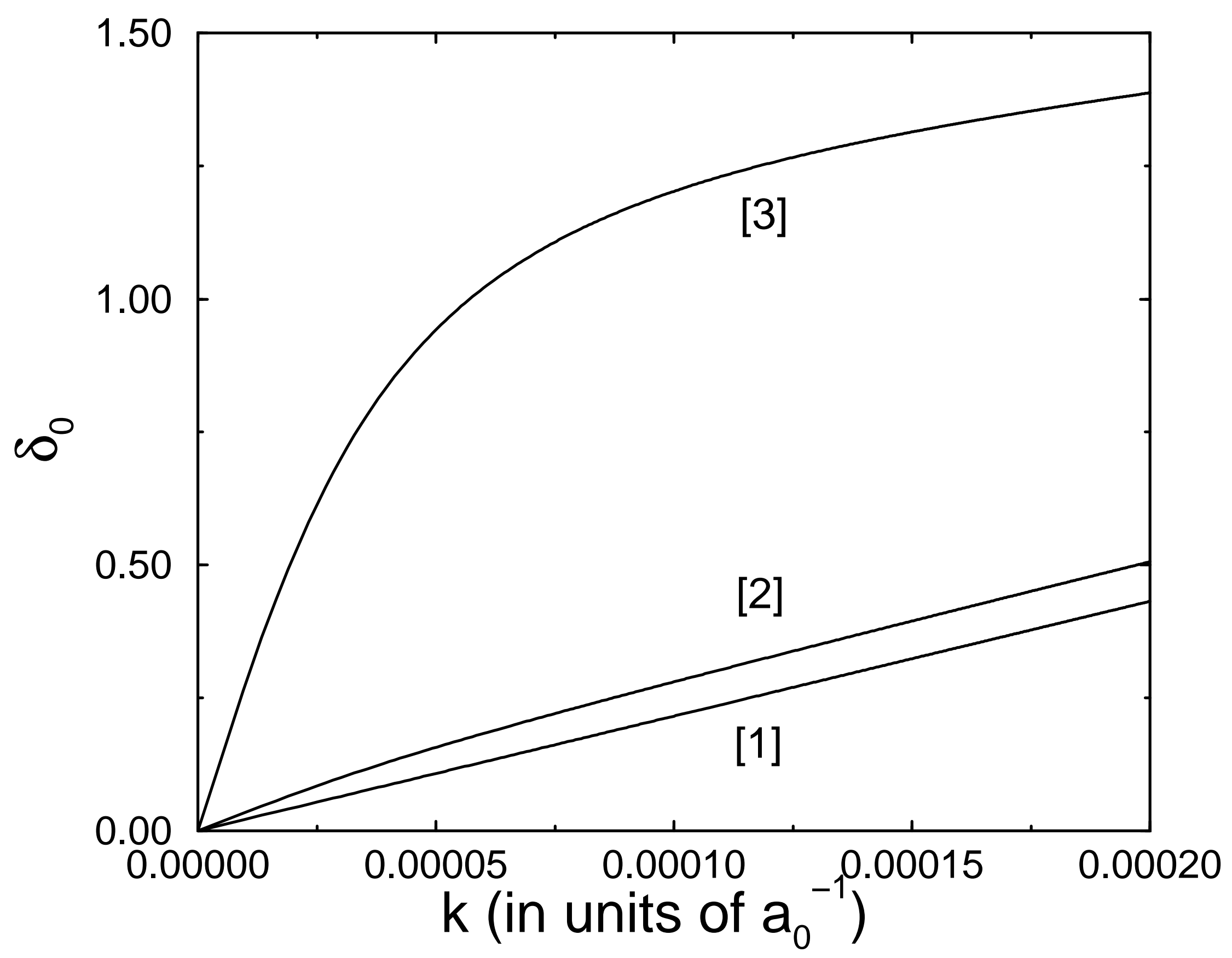


M.J.Bijlsma et al. fig.3

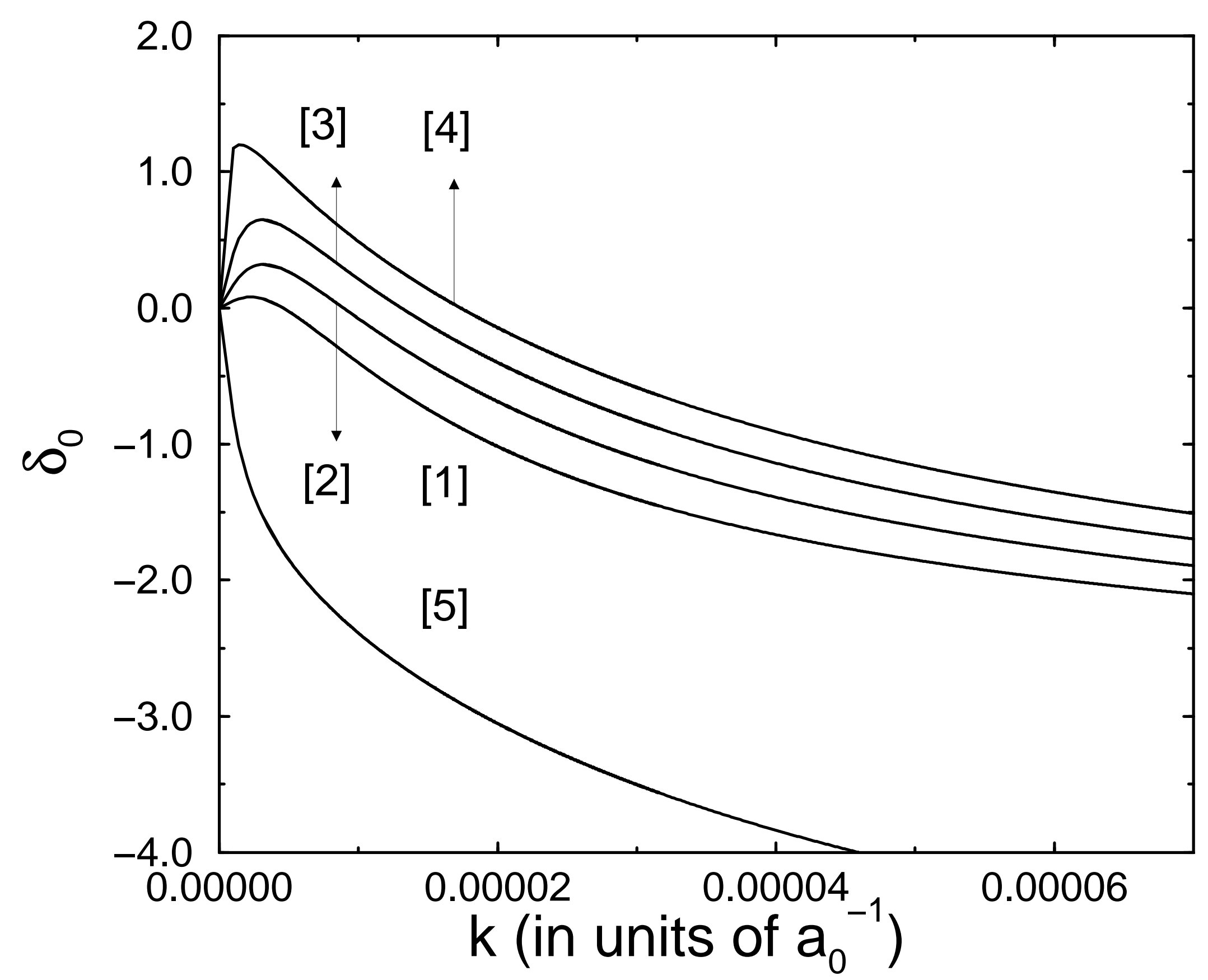


M.J.Bijlsma et al. fig.4

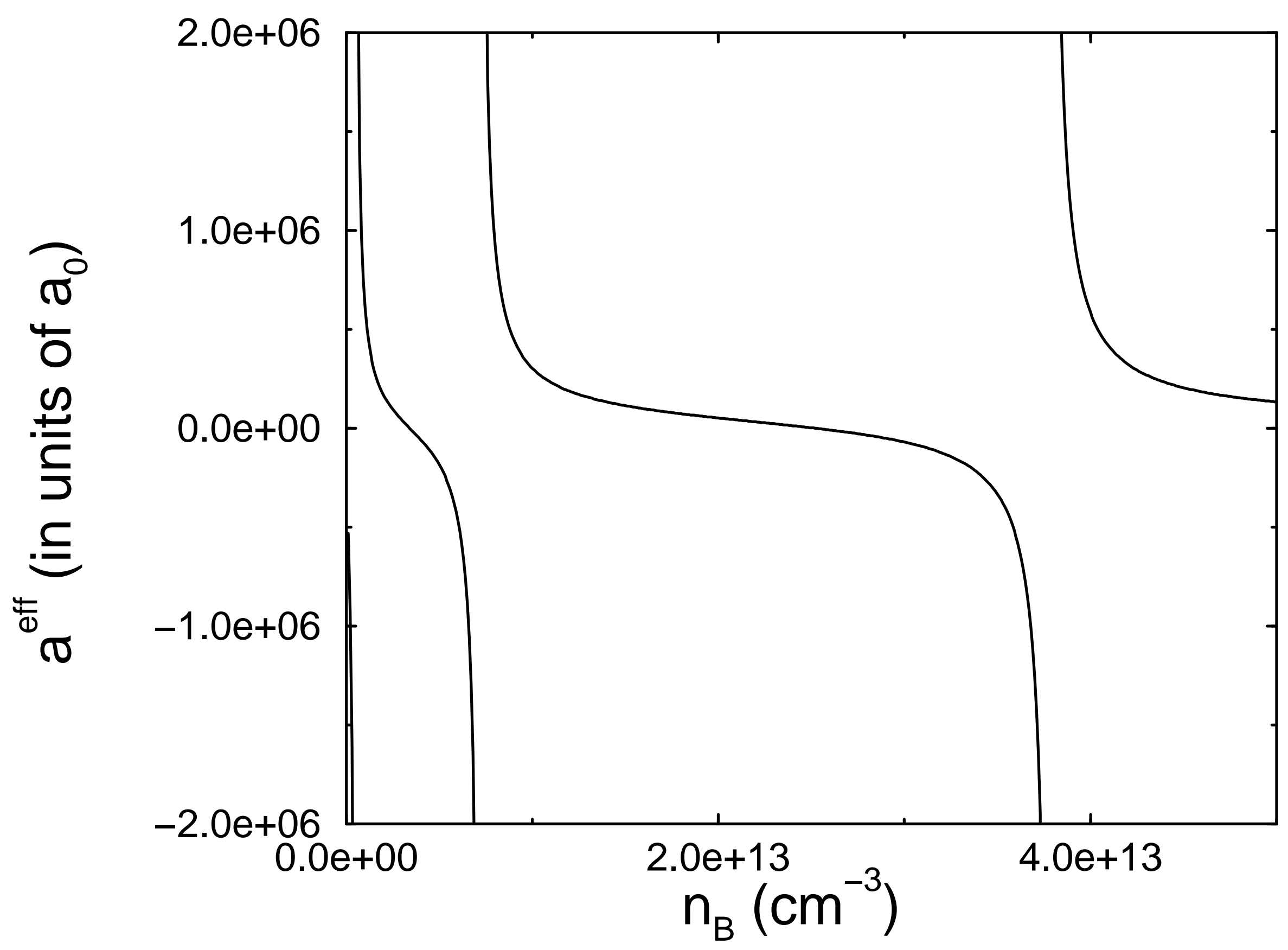

